\title{
La prueba del polígrafo: Una dinámica de aprendizaje basado en juegos (Game Based Learning, GBL) aplicada al estudio de la Fisiología Humana.
}

\author{
Úrsula Muñoz ${ }^{\mathrm{a}}$, Asier $\mathrm{Jayo}^{\mathrm{b}}, \mathrm{M}^{\mathrm{a}}$ de la Cruz Sádaba ${ }^{\mathrm{c}}$, Rima Barhoum ${ }^{\mathrm{e}}$, Esther \\ Escudero $^{\text {d, Isabel Sánchez-Vera }}{ }^{\mathrm{f}}$.
}

aursula.munozmoron@ceu.es, ${ }^{b}$ asier.jayoandres@ceu.es, cmariacruz.sadabaargaiz@ceu.es, ${ }^{d}$ estheresc@,ceu.es, ${ }^{\text {rrbarho@,ceu.es, } \quad \text { fisanver@ceu.es }}$ Todos los autores tienen la misma procedencia: Sección de Fisiología. Dpto. Ciencias Médicas Básicas. Facultad de Medicina. Universidad San Pablo CEU, Madrid.

\begin{abstract}
:
The polygraph test is a practice that we perform for the study of sympathetic tone as a physiological response to cognitive and emotional stimuli. During previous courses we identified some difficulties that limit their educational scope, such as the lack of involvement of the students, both to perform the test and to achieve a conclusion after the interpretation of the results. We aimed to create an environment to generate intrinsic motivation and involvement of the students, so that the acquisition of theoretical concepts and the performance of the experimental methodology, based on the scientific method, were an entertaining, dynamic and active process. In order to overcome the difficulties observed in previous courses, we carried out an approach of Game Based Learnig (GBL) and role-playing by adapting a version of the Cluedo game ("The Mayor has been murdered!"). This method improved significantly the skills reached by the students compared to the method used previously. Moreover, the assessment of the activity by the students and teachers was very positive. In summary, we consider that the experience meets our preliminary aims, so we will carry out it in the upcoming courses.
\end{abstract}

Keywords: Game Based Learning (GBL), role-playing, intrinsic motivation, specific skills, collaborative learning.

\section{Resumen}

La prueba del polígrafo es una práctica que realizamos para promover el estudio de la respuesta simpática como adaptación fisiológica a estímulos cognitivos y emocionales. Durante varios cursos hemos podido identificar una serie de dificultades relacionados con la falta de implicación de los alumnos, tanto para realizar la prueba, como para llegar a una conclusión tras el análisis de los resultados. Nuestro objetivo principal en este caso fue crear un entorno que generara de forma intrínseca motivación e implicación de los alumnos, de forma que la adquisición de conceptos teóricos y la metodología experimental basada en el método científico constituyese un proceso entretenido, dinámico y activo. A fin de superar las dificultades observadas en cursos previos, realizamos una estrategia de aprendizaje basado en juegos (Game Based Learning, GBL) y adquisición de roles dentro de nuestro modelo inicial adaptando una versión del juego del Cluedo (“¡Han asesinado al alcalde!”). De esta forma, conseguimos mejorar significativamente las competencias alcanzadas por los alumnos que realizaron la práctica sin la nueva dinámica, y la valoración de la actividad por 
parte del alumnado y profesorado fue muy positiva. En conjunto, consideramos que la experiencia cumple con nuestros objetivos preliminares, por lo que seguiremos desarrollándola en los cursos venideros.

\section{Palabras clave: Aprendizaje basado en juegos, adquisición de roles, motivación intrínseca, competencias especificas, trabajo colaborativo.}

\section{Introducción}

Uno de los retos que se nos plantea como docentes es aumentar la motivación intrínseca de los estudiantes; es decir, lograr su implicación en el proceso de aprendizaje por placer o interés y no por la calificación final (Rian \& Deci, 2000). La incorporación de juegos en las actividades docentes proporciona un feedback inmediato, control sobre el material didáctico, y una curiosidad inspiradora que satisface estas necesidades intrínsecas (Kapp, 2012). Esta motivación intrínseca esta fundamentada principalmente en la obtención de "recompensas" de naturaleza diversa que se establecen al comienzo de la dinámica. Este tipo de aproximaciones favorecen el compromiso y la implicación de los alumnos, promueven el trabajo en equipo y además posibilitan que el proceso de aprendizaje sea divertido mejorando los procesos de aprendizaje y sus resultados (Shute \& Ventura, 2013) todo ello nos aproxima a un modelo de aprendizaje más proactivo (Dias, 2017).

Para conseguir establecer un modelo de aprendizaje más activo, los profesores de la sección fisiología llevamos varios años implantando técnicas de innovación en nuestras aulas (Escudero et al. 2017, Sánchez-Vera et al. 2017, Escudero et al. 2018, Muñoz et al. 2018). En este contexto de innovación, desde hace varios cursos estamos utilizando el polígrafo para aproximarnos al estudio de la regulación del Sistema Nervioso Autónomo y la activación del tono simpático en seres humanos. Esta actividad se lleva a cabo en las prácticas de la asignatura Fisiología Humana II, que se imparte en el segundo cuatrimestre del $2^{\circ}$ curso del grado de Medicina.

El objetivo inicial de la práctica era estudiar un concepto propio del currículo de Fisiología Humana (Regulación del Sistema Nervioso Autónomo) y que nuestros alumnos adquiriesen ciertas competencias específicas, como conocer en qué situaciones se activa el Sistema Nervioso Simpático, ser capaz de realizar pruebas funcionales y/o determinar parámetros vitales e interpretarlos. Asi mismo, también nos interesaba que adquiriesen ciertas competencias transversales, como reunir e interpretar datos fisiológicos relevantes de forma que fuesen capaces de emitir juicios que incluyesen una reflexión previa. Para posibilitar la adquisición de las mismas, incialmente, y durante varios cursos contextualizamos a nuestros alumnos en un entorno en el que el registro de las constantes vitales de sus compañeros se realizarían con la finalidad de someterles a "La prueba del polígrafo" o "Prueba de la verdad". De esta manera, los alumnos tendrían que descubrir si las variaciones observadas en las constantes de sus compañeros podrían ser indicativas de que estuvieran mintiendo. Contextualizar a los alumnos en un entorno de juego inicial en el que tenían que ser capaces de descifrar quién y cuándo estaba mintiendo favorecía de forma relevante su disposición al trabajo propuesto. 
La realización de esta práctica con alumnos voluntarios que se convertían en sujetos de "interrogatorios" planteaba dos dificultades importantes: por un lado, la participación de los alumnos como sujetos de estudio está muy condicionada por la posibilidad de sentirse expuesto en una prueba de este tipo, lo que limita en gran medida la participación. Por otro lado, la variabilidada en los sujetos de estudio y el tipo de interrogatorio generado, complicabla interpretación de resultados, y por lo tanto la adquisición de las competencias transversales propuestas para la presente dinámica.

Para resolver estas dificultades, el presente curso nos planteamos incluir una segunda modificación al modelo inicial que incluyese la adquisición de una serie de roles basados en una interpretación de la adaptación del popular juego del Cluedo ("Han asesinado al alcalde de Madrid”, Martín Ruiz, 2010). Esta adaptación a nuestra propuesta inicial, comprende por tanto un juego basado en la adquisición de roles o role-playing: En ella, el alcalde de Madrid ha sido asesinado, por lo que los alumnos deben de ser capaces de identificar al asesino utilizando el polígrafo con los distintos sospechosos. Las normas se establecen al comienzo de la práctica, de forma que se genera un entorno propicio para el juego, los alumnos preparan su estrategia, y la propia dinámica genera la "recompensa" (descubir al asesino, o no ser descubierto). Así, la técnica del polígrafo pasa en esta nueva aproximación de ser un fin en sí mismo, a ser la herramienta para la adquisición de conocimientos formales (competencias) e informales (atrapar al asesino y por tanto, ganar el juego).

Estas técnicas de simulaciones, en las que los alumnos desempeñan un papel concreto en el contexto de una situación simulada siguiendo determinadas reglas en las que interaccionan entre ellos, se ha visto que conllevan a una mejora en el proceso de aprendizaje del propio estudiante, facilita la interacción entre ellos el trabajo colaborativo; y en definitiva, un aprendizaje más proactivo (Ruben, 1999). La evaluación de la adquisición de las competencias antes descritas se hizo mediante el uso de rúbricas. Ambos tipos de competencias están incluidas en la guía docente de la asignatura, y se ajustan al plan de Bolonia que transformó los programas educativos por objetivos a programas por competencias, dándole un papel protagonista al alumno durante el proceso de aprendizaje (De la Cruz, 2005).

\section{Objetivo}

El principal objetivo que pretende esta actividad es crear un entorno que genere de forma intrínseca motivación e implicación de los alumnos, de forma que la adquisición de conceptos teóricos y de la metodología experimental basada en el método científico constituya un proceso entretenido, dinámico y activo. De esta manera, se pretende que los alumnos integren las competencias descritas previamente como una herramienta útil para la resolución de un problema ficticio planteado en un contexto propio de un juego.

\section{Desarrollo de la innovación}

\subsection{Descripción de los grupos.}

Esta aproximación se realizó con alumnos del $2^{\circ}$ curso de Medicina, durante una clase práctica de tres horas de duración, en la asignatura de Fisiología Humana II (segundo 
semestre). Los resultados se generaron a partir del análisis de dos cursos en los se han realizado la misma práctica, pero en los que se han seguido dos dinámicas diferentes. En el curso anterior (2017-18) se realizó la prueba del polígrafo sin la dinámica del Cluedo, al que llamamos Grupo 1. Y en el curso actual, con la dinámica del Cluedo, al que llamamos Grupo 2 (2018-19). En ambos cursos el número total de alumnos que participó en la actividad fue de 80. Se dividieron en grupos de práctica en los que el número de alumnos no fue superior a 12. Los profesores que participaron en la práctica y los horarios en la que se impartieron fueron los mismos para ambos grupos.

\subsection{Descripción de la actividad:}

Para la realización de la presente actividad, los alumnos contaban con:

I. Un guion explicativo con los fundamentos teórico-prácticos necesarios para la realización de la actividad y una ficha para que rellenaran el día de la práctica. (disponible con anterioridad a la misma).

II. Una explicación oral "in situ" por parte del docente en el que se explicaron los fundamentos teórico-prácticos ya disponibles en el guion, e indicaciones de la dinámica a seguir utilizando medios audiovisuales.

III. El material técnico necesario para la prueba del Polígrafo (Biopac 35SL): neumógrafo para el registro de la frecuencia respiratoria, electrodos para el registro de la frecuencia cardiaca y para el registro de la actividad electrodermal.

Con el objetivo de superar las dificultades observadas en cursos previos, realizamos una estrategia de aprendizaje basada en la adquisición de roles o role-playing adaptando una versión del juego del Cluedo (“¡Han asesinado al alcalde!”). La dinámica del cluedo se detalla a continuación:

1. Han asesinado al alcalde de Madrid y la policía ha arrestado a tres sospechosos.

2. Se divide a la clase entre sospechosos y policías, de forma que tres alumnos vountarios adoptaran el rol de distintos sospechosos propuestos en el juego del cluedo.

3. Los sospechosos salen de la clase y se les informa sobre las circunstancias "reales" del asesinato, quién fue el asesino y sus respectivas coartadas.

4. En paralelo, el resto de la clase diseña el interrogatorio específico para cada uno de los sospechosos, a fin de poder acertar quién fue el culpable.

Una vez preparados los personajes por los alumnos voluntarios y el interrogatorio por el resto de la clase, los alumnos realizan el interrogatorio a cada uno de los sospechosos con la ayuda del polígrafo.

Para realizar la prueba se llevaron a cabo los siguientes pasos:

a) Monitorización de variables fisiológicamente relacionadas: a cada sospechoso los alumnos le colocaron distintos sensores para medir las siguientes variables: la frecuencia cardiaca, la frecuencia respiratoria y la actividad electrodermal. 


\section{b) Registro de las variables anteriores en respuesta a:}

1. Preguntas de lógica (Sin implicación emocional, pero si cognitiva)

2. Respuesta a los colores (respuesta emocional frente a un estímulo sensorial)

3. Preguntas control (con respuesta conocida)

4. Interrogatorio con preguntas específicas (previamente elaboradas por ellos mismos).

c) Interpretación de los resultados. Análisis de los resultados cualitativos y cuantitativos de cada una de las pruebas. Posteriormente se debatieron los resultados más reseñables tratando de identificar al supuesto asesino.

\subsection{Evaluación de la adquisición de competencias:}

Todos los datos obtenidos en la práctica fueron recogidos por los alumnos en una ficha de seguimiento para evaluar si se habían conseguido las siguientes competencias:

1. Adquirir y registrar los valores de actividad electrodermal, ritmo cardiaco y ritmo respiratorio obtenidos durante la realización de la prueba.

2. Analizar los resultados obtenidos.

3. Contestar preguntas relacionadas con el tono simpático y su activación.

A estos efectos, se establecieron 4 categorías diferentes para cada una de las competencias siguiendo el modelo de Babiera Puig (Babiera Puigy et al. 2017):
A. Excelente
B. Óptimo.
C. Puede mejorar
D. No alcanzado.

Con el fin de facilitar una media por grupo, estas 4 categorías se han traducido a números siguiendo la siguiente escala: $\mathrm{A}=3, \mathrm{~B}=2, \mathrm{C}=1$ y $\mathrm{D}=0$.

Al finalizar el bloque de prácticas se pasaron dos encuestas anónimas dirigidas a personal docente y alumnado implicado en la práctica, utilizando el portal web del CEU y la plataforma Socrative.

Los docentes valoraron el nivel de atención, participación e implicación de los alumnos. Y los alumnos valoraron la experiencia según los siguientes apartados:

$>$ Entretenimiento e interés

$>$ Utilidad y aprendizaje

$>$ Atención y seguimiento

$>$ Preferencia frente a otro tipo de dinámicas

$>$ Valoración final de la dinámica 
La prueba del poligrafo: Una dinámica de aprendizaje basado en juegos (Game Based Learning, GBL) aplicada al estudio de la fisiología humana.

\subsection{Análisis de datos:}

Para ver si había diferencias significativas entre ambos grupos se utilizó como test estadístico la prueba Ji cuadrado $\left(\chi^{2}\right)$ de Pearson. Los resultados que obtuvimos para cada una de las competencias se muestran a continuación.

\section{Resultados}

\subsection{Adquirir y registrar los valores de actividad electro dermal, ritmo cardiaco y ritmo respiratorio obtenidos durante la realización de la prueba.}

En ambos grupos todos los alumnos tuvieron puntuación, no estando ninguno de ellos en la categoría de no alcanzado. Para esta competencia la mayoría obtuvieron la máxima calificación, como podemos observar en la Tabla1. La media total fue mayor en el Grupo 2 $(2,93)$ que en el Grupo $1(2,7)$.

\begin{tabular}{|c|c|c|c|}
\hline \multicolumn{2}{|c|}{ Tabla1. Adquisición de datos } \\
\hline $\begin{array}{c}\text { Número de } \\
\text { Alumnos }\end{array}$ & Grupo 1 & Grupo 2 & Total \\
\hline $\begin{array}{c}\text { No alcanzado } \\
\text { (0 puntos) }\end{array}$ & 0 & 0 & 0 \\
\hline $\begin{array}{c}\text { Puede mejorar } \\
\text { (1 punto) }\end{array}$ & 5 & 1 & 6 \\
\hline $\begin{array}{c}\text { Óptimo } \\
\text { (2 puntos) }\end{array}$ & 14 & 4 & 18 \\
\hline $\begin{array}{c}\text { Excelente } \\
\text { (3 puntos) }\end{array}$ & 61 & 75 & 136 \\
\hline Total & 80 & 80 & 160 \\
\hline
\end{tabular}

El porcentaje de alumnos que obtuvieron la máxima calificación para esta competencia fue mayor en el Grupo 2 que en el Grupo 1; siendo esta diferencia estadísticamente significativa, como se puede observar en la figura 1. 


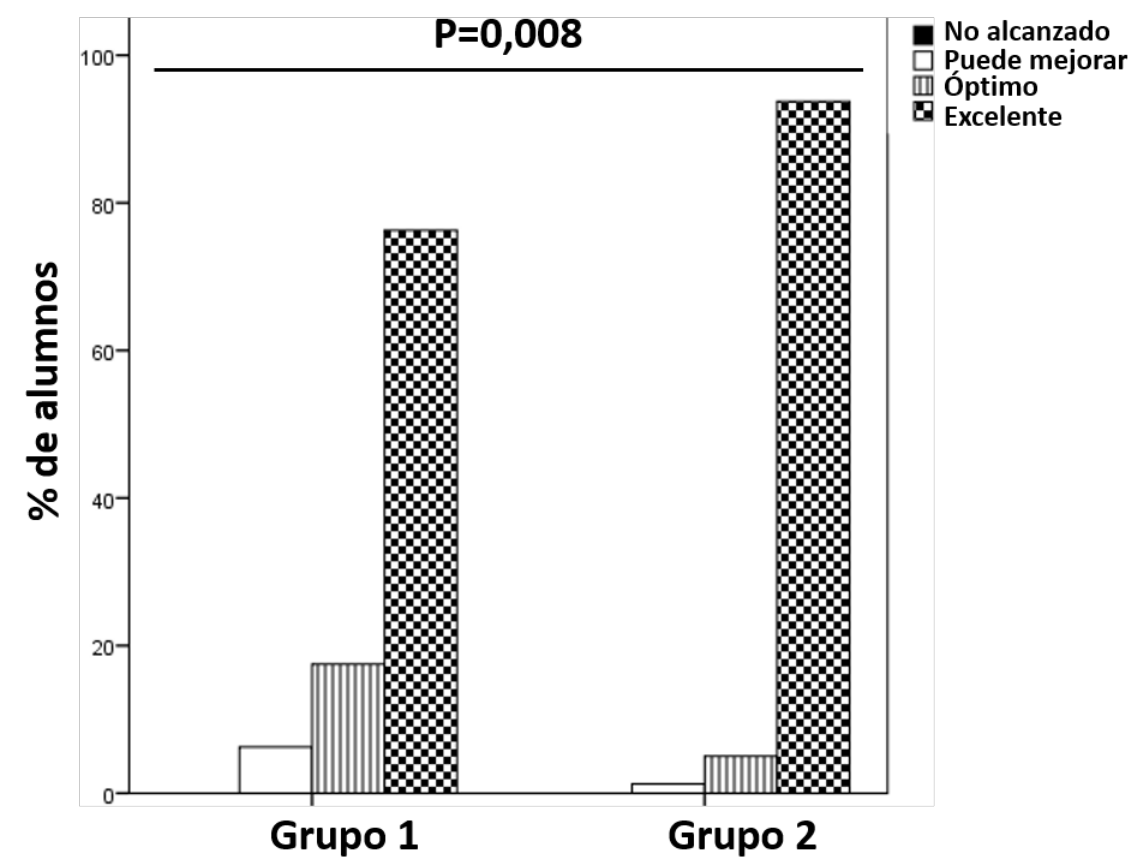

Figura 1. Porcentaje de alumnos con distintas puntuaciones para la competencia de adquisición de datos. El grupo 1 representa a los alumnos que realizaron la práctica sin la dinámica del cluedo. El grupo número 2 representa a los alumnos que sí incluyeron la dinámica del cluedo. Las barras representan el porcentaje de alumnos que no alcanzan, que pueden mejorar, que alcanzan un nivel óptimo y que alcanzan un nivel excelente en la competencia evaluada.

\subsection{Analizar los resultados obtenidos.}

En esta competencia un número muy bajo de alumnos no puntuaron en ambos grupos. En el Grupo 1 la mayor parte de los alumnos se encuentran entre las puntuaciones 1 punto (puede mejorar) y 2 puntos (óptimo); sin embargo, la mayoría de los alumnos de Grupo 2 obtuvieron la máxima calificación: 3 puntos (excelente). La media total fue mayor en el grupo $2(2,81)$ que en el grupo $1(1,95)$. Ver valores en la Tabla 2.

\begin{tabular}{|c|c|c|c|}
\hline \multicolumn{3}{|c|}{ Tabla 2. Análisis de datos } & \\
\hline $\begin{array}{c}\text { Número de } \\
\text { Alumnos }\end{array}$ & Grupo 1 & Grupo 2 & Total \\
\hline $\begin{array}{c}\text { No alcanzado } \\
\text { (0 puntos) }\end{array}$ & 6 & 1 & 7 \\
\hline $\begin{array}{c}\text { Puede mejorar } \\
\text { (1 punto) }\end{array}$ & 16 & 3 & 19 \\
\hline $\begin{array}{c}\text { Óptimo } \\
(2 \text { puntos })\end{array}$ & 34 & 6 & 40 \\
\hline $\begin{array}{c}\text { Excelente } \\
(3 \text { puntos) }\end{array}$ & 24 & 70 & 94 \\
\hline Total & 80 & 80 & 160 \\
\hline
\end{tabular}


El porcentaje de alumnos que obtuvieron la máxima calificación para esta competencia fue mayor en el Grupo 2 que en el Grupo 1; siendo esta diferencia estadísticamente significativa, como se puede observar en la figura 2.

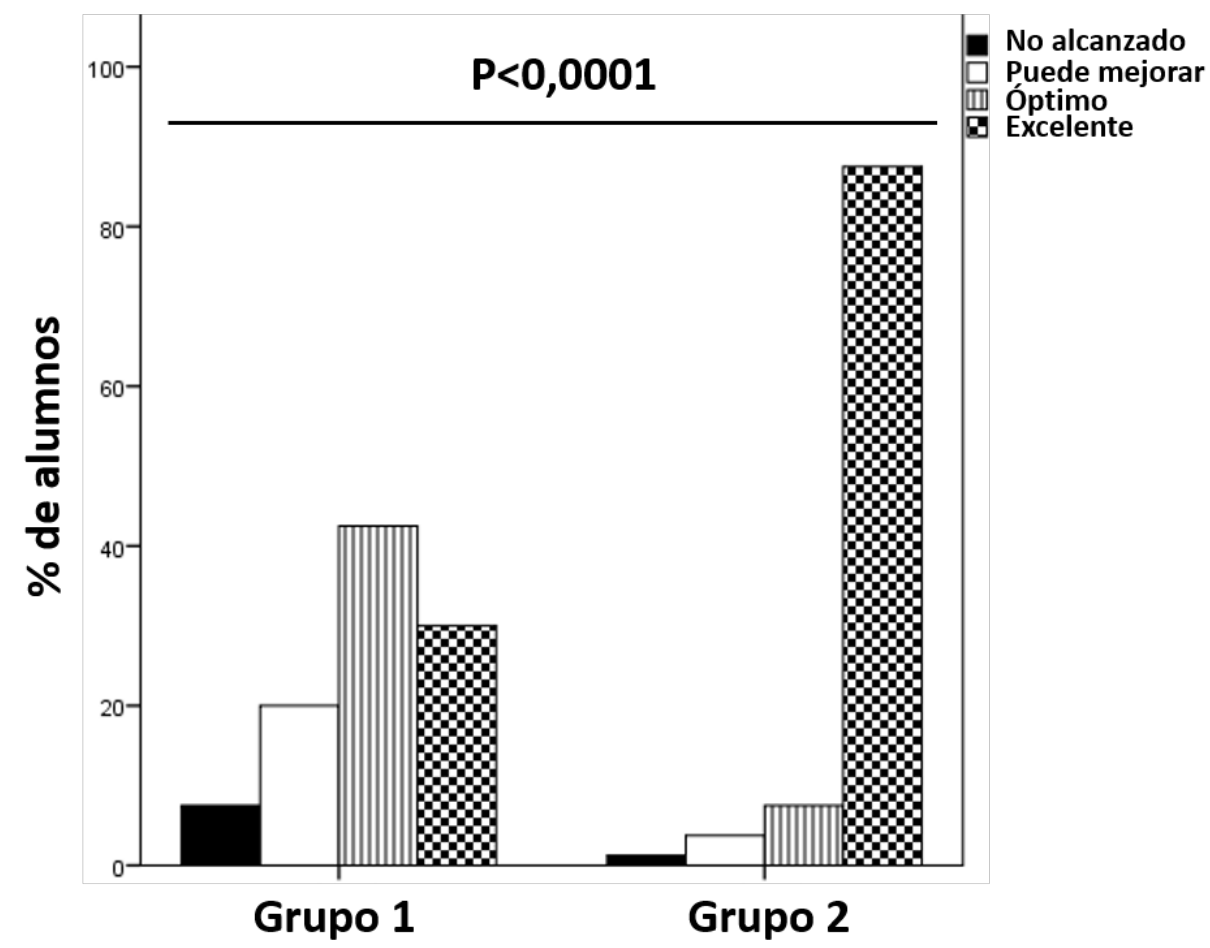

Figura 2. Porcentaje de alumnos con distintas puntuaciones para la competencia de análisis de datos. El grupo 1 representa a los alumnos que realizaron la práctica sin la dinámica del cluedo. El grupo número 2 representan a los alumnos que si incluyeron la dinámica del cluedo. Las barras representan el porcentaje de alumnos que no alcanzan, que pueden mejorar, que alcanzan un nivel óptimo y que alcanzan un nivel excelente en la competencia evaluada.

\subsection{Contestar preguntas relacionadas con el tono simpático y su activación.}

Los resultados para esta última competencia variaron poco entre ambos grupos (Tabla 3). La mayoría de los alumnos obtuvieron la máxima calificación variando la media total muy poco entre ambos grupos, en el grupo 2 fue de 2,8 mientras que en el grupo 1 estaba en torno a 2,7. Esta ligera diferencia no fue estadísticamente significativa, como se puede apreciar en la figura 3. 


\begin{tabular}{|c|c|c|c|}
\hline Tabla 3. Preguntas sobre la actividad \\
\hline $\begin{array}{c}\text { Número de } \\
\text { Alumnos }\end{array}$ & Grupo 1 & Grupo 2 & Total \\
\hline $\begin{array}{c}\text { No alcanzado } \\
\text { (O puntos) }\end{array}$ & 0 & 0 & 0 \\
\hline $\begin{array}{c}\text { Puede mejorar } \\
\text { (1 punto) }\end{array}$ & 4 & 1 & 5 \\
\hline $\begin{array}{c}\text { Óptimo } \\
(2 \text { puntos) }\end{array}$ & 19 & 11 & 30 \\
\hline $\begin{array}{c}\text { Excelente } \\
\text { (3 puntos) }\end{array}$ & 57 & 68 & 125 \\
\hline Total & 80 & 80 & 160 \\
\hline
\end{tabular}

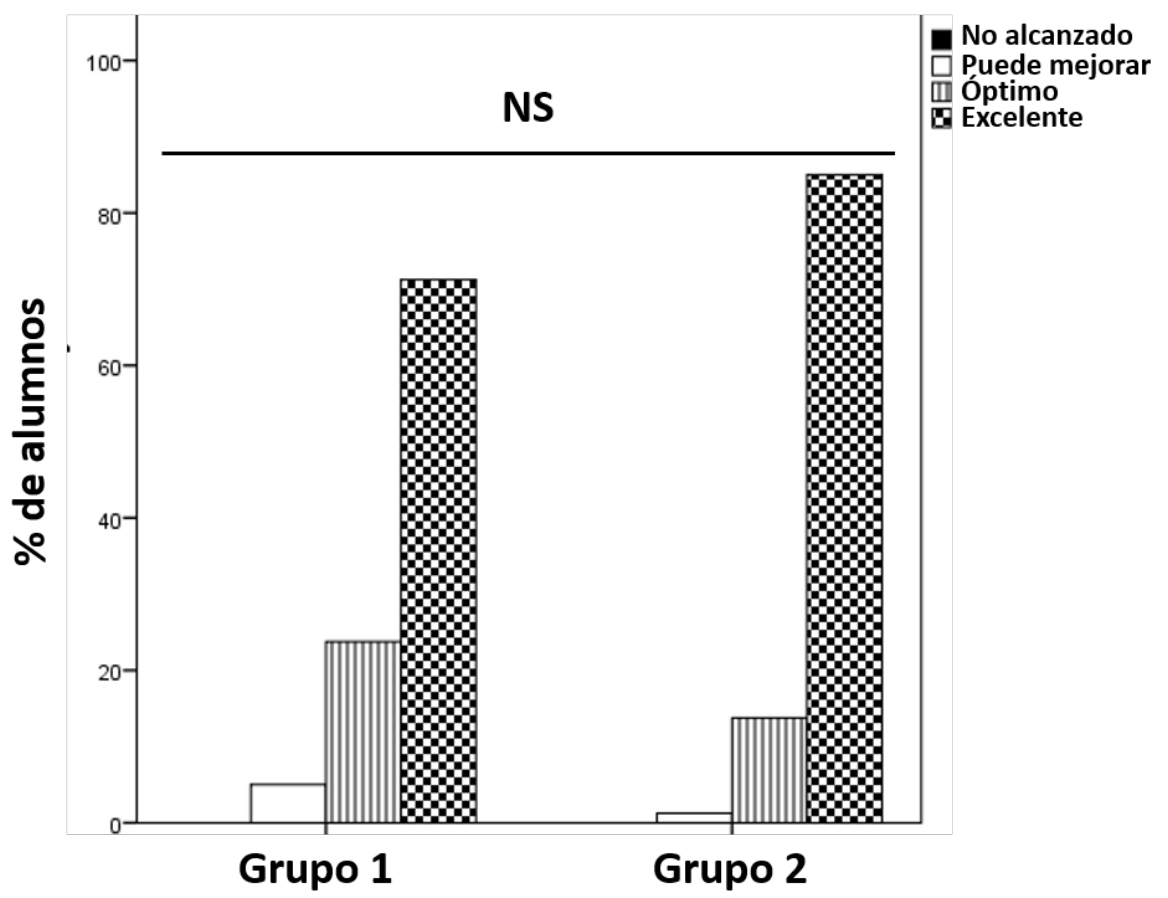

Figura 3. Porcentaje de alumnos con distintas puntuaciones para la competencia conocimientos sobre el tono simpático. El grupo 1 representa a los alumnos que realizaron la práctica sin la dinámica del cluedo. El grupo número 2 representan a los alumnos que si incluyeron la dinámica del cluedo. Las barras representan el porcentaje de alumnos que no alcanzan, que pueden mejorar, que alcanzan un nivel óptimo y que alcanzan un nivel excelente en la competencia evaluada.

\subsection{Valoración de la nueva dinámica por profesores y alumnos}

Los resultados de la experiencia se valoraron a través de dos tipos de encuestas anónimas dirigidas a docentes y alumnado. Los docentes valoraron de forma muy positiva la nueva dinámica, tanto en términos generales como a nivel de atención, participación e implicación de los alumnos. Asimismo, consideraron que la presente dinámica presentaba ventajas con respecto a la anterior. 
Para valorar la acogida de los alumnos a la dinámica del cluedo, se les paso una encuesta con 5 preguntas. En la encuesta participaron el 100\% de los alumnos que realizaron la práctica. Los resultados de las preguntas de la encuesta se muestran a continuación.

En la figura 4 se recoge los resultados de la primera pregunta de la encuesta donde se preguntó qué opinión les merecía la dinámica del cluedo durante la práctica del polígrafo. La mayor parte de los alumnos, el 95\%, contestaron que muy entretenida o entretenida, solo un $5 \%$ opinaran regular o aburrida.

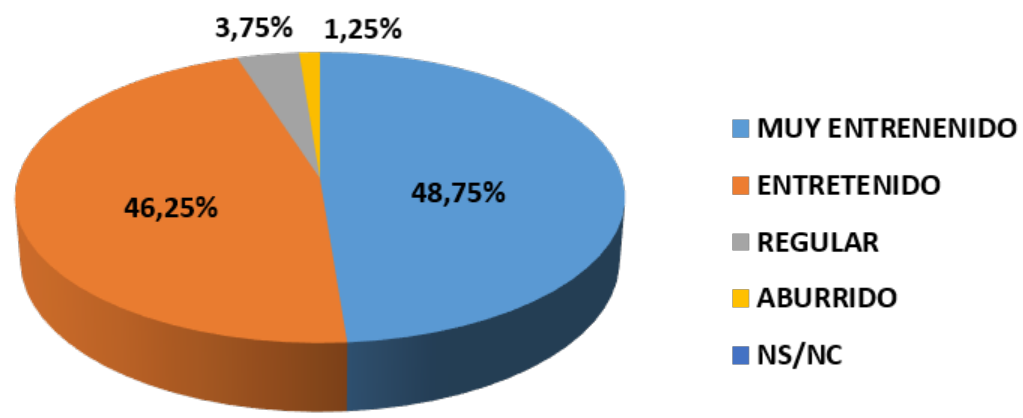

\section{Figura 4. Entretenimiento e interés}

La siguiente pregunta fue sobre la utilidad de la dinámica a la hora de comprender el proceso del interrogatorio y la técnica del polígrafo. La mayoría de los alumnos contestaron que muy útil o útil. Solo un porcentaje bajo contestó regular o poco útil (figura 5).

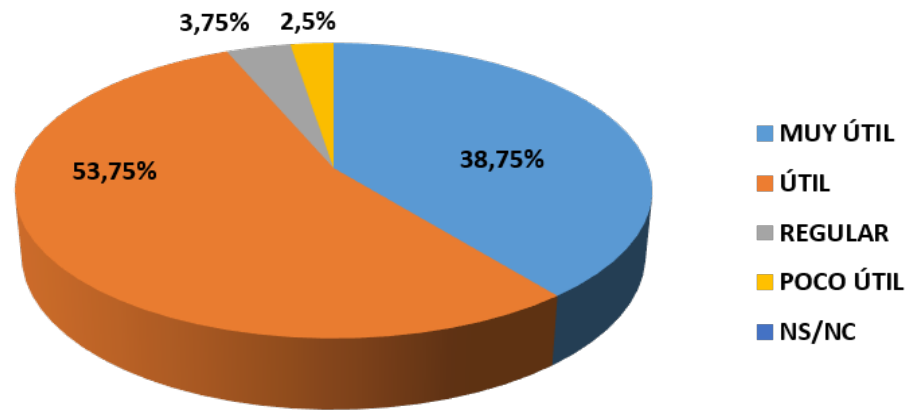

Figura 5. Utilidad y aprendizaje

Respecto a la pregunta de si les ayudo la dinámica del cluedo a seguir el protocolo propuesto en la práctica. Un $67,5 \%$ contesto que, en gran medida, un $30 \%$ en menor medida y para un 2,5\% les fue indiferente o contraproducente (figura 6). 


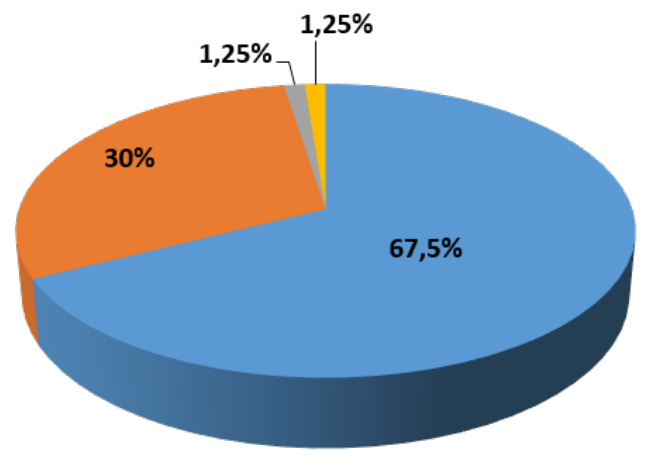

\author{
- SÍ, EN GRAN MEDIDA \\ SI, EN MENOR MEDIDA \\ NO, FUE INDIFERENTE \\ $\square$ NO, FUE \\ CONTRAPRODUCENTE \\ nS/NC
}

\title{
Figura 6. Atención y seguimiento
}

Para finalizar, quisimos conocer la preferencia de los alumnos sobre el uso de la dinámica del cluedo (en la que se utilizan personajes ficticios) o si por el contrario preferirían otra dinámica, como por ejemplo, realizar la prueba del polígrafo entre ellos mismos con preguntas personales. Sorprendentemente el $38,75 \%$ piensa que sería más entretenido empatados con aquellos que piensan que no se centrarían en la práctica o les resultaría incómodo (figura 7).
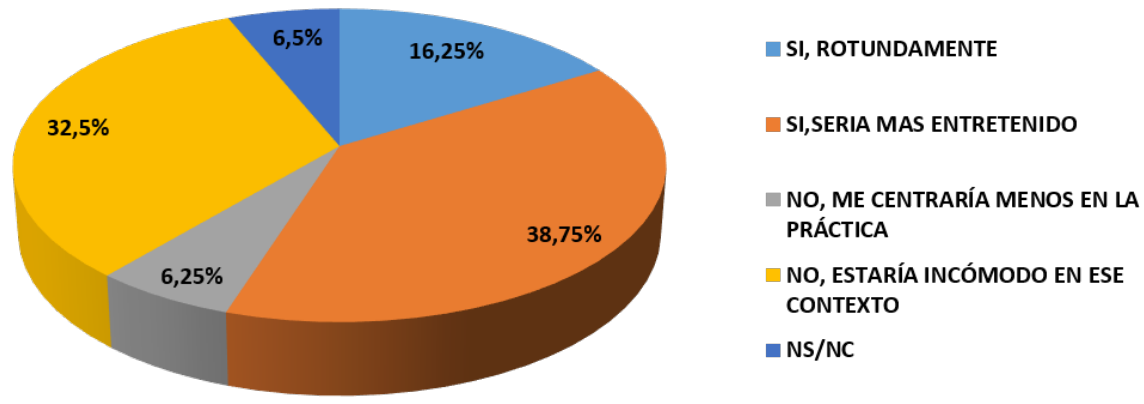

\section{Figura 7. Preferencia frente a otro tipo de dinámica.}

Por último, le preguntamos sobre la utilidad de juego del cluedo para el funcionamiento y transcurso de la práctica, la gran mayoría opinaron que hace que sea más dinámica (figura 8). 


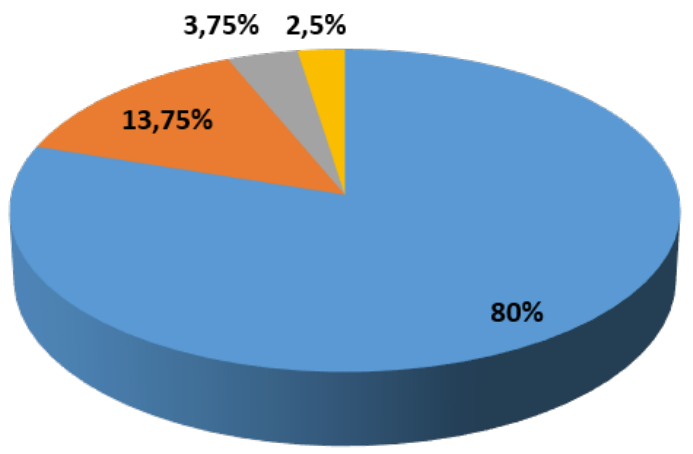

\author{
SÍ, HACE QUE SEA MÁS \\ DINÁMICA \\ SÍ, PERO SÓLO PARA EL \\ PRIMER INTERROGATORIO \\ NO, CREO QUE NO MÁS QUE \\ OTRA DINÁMICA \\ NO, CREO QUE ES \\ CONTRAPRODUCENTE \\ NS/NC
}

Figura 8. Valoración de la actividad

\title{
6. Conclusiones.
}

Podemos sacar tres conclusiones importantes tras la actividad:

La práctica del polígrafo en su configuración actual es útil para comprender la actividad del Sistema Nervioso Simpático sobre varios parámetros fisiológicos.

La introducción de la segunda dinámica de aprendizaje basado en juegos con reparto especifico de roles contribuyó a mejorar significativamente las competencias alcanzadas por los alumnos, la atención y la participación, al mismo tiempo que generaba un entorno seguro.

Nuevas estrategias de aprendizaje muy sencillas pueden aportar un valor intrínseco añadido a la práctica, las cuales favoreceren la atención, participación y adquisición de conocimientos.

En conjunto, y en base a nuestros objetivos, consideramos que la experiencia ha resultado muy positiva.

\section{Referencias:}

1. RYAN, RM \& DECI, EL (2000). "Intrinsic and extrinsic motivations: Classic definitions and new directions". Contemporary Educational Psychology, vol. 25, p. 54-67.

2. KAPP, K (2012). "The gamification of learning and instruction: gamebased methods and strategies for training and education". San Francisco, CA: Pfeifle

3. SHUTE, VJ \& VENTURA, M (2013). "Stealth assessment: Measuring and supporting learning in games". Cambridge, MA: Massachusetts Institute of Technology Press Books. 
4. DIAS, J (2017). Teaching operations research to undergraduate management students: The role of gamification. The International Journal of Management Education, 15, 98-111.

5. ESCUDERO, E., SÁNCHEZ-VERA, I., BARHOUM, R., MUÑOZ, U \& JAYO, A (2018). Análisis de la metodología Flipped learnig en el entorno de la práctica de la Fisiología Médica.

Doi: http://dx.doi.org/10.4995/INRED2018.2018.8616.

6. SÁNCHEZ-VERA, I., ESCUDERO, E., MUÑOZ, U., BORREGO, MJ \& BARHOUM, R (2017). Experiencia en la elaboración de videos didácticos por alumnos de Fisiología como parte de su proceso de aprendizaje.

Doi: http://dx.doi.org/10.4995/INRED2017.2017.6819

7. ESCUDERO, E., SÁNCHEZ-VERA, I., BARHOUM, R., PUCHE, JE \& MUÑOZ, U (2017). Análisis del uso de autoevaluaciones en una plataforma digital en el entorno de la Fisiología Médica.

Doi: http://dx.doi.org/10.4995/INRED2017.2017.6809.

8. MUÑOZ, U., ESCUDERO, E., BARHOUM, R., SÁDABA, MC \& SÁNCHEZVERA, I (2018). Generación colaborativa de conocimiento. Una experiencia de aprendizaje basada en equipos o Team Based Learning (TBL) en la práctica odontológica.

Doi: http://dx.doi.org/10.4995/INRED2018.2018.8723

9. DE LA CRUZ, MA (2005): taller sobre el proceso de aprendizaje-enseñanza de competencias. Zaragoza:instituto de ciencias de la Educación de la Universidad de Zaragoza.

10. MARTÍN RUIZ, G (2010). “¡Han asesinado al alcalde! Un juego para la clase de E/LE. "redELE: Revista Electrónica de Didáctica ELE, ISSN-e 1571$4667, \mathrm{~N}^{\circ} .19$.

11. RUBEN, B.D (1999). Simulations, Games, and Experiende Based Learning: The quest for a New Paradigm for teaching. Simulation and gaming. 30 (4), 498-505.

12. BAVIERA-PUIG, A., ESCRIBA-PEREZ, C \& BUITRAGO-VERA, J (2017). Estrategias de evaluación para las competencias transversales CT-02, CT-04 y CT-06. Doi:http://dx.doi.org/10.4995/INRED2017.2017.6669 